\title{
The bacterial microbiota in the oral mucosa of rural Amerindians
}

\begin{abstract}
Correspondence
Maria G. Dominguez-Bello maria.dominguez1@upr.edu
\end{abstract}

Received 1 July 2010

Revised 6 September 2010

Accepted 14 September 2010

\author{
Monica Contreras, ${ }^{1}$ Elizabeth K. Costello, ${ }^{2}$ Glida Hidalgo, ${ }^{3}$ Magda Magris, ${ }^{3}$ \\ Rob Knight ${ }^{2,4}$ and Maria G. Dominguez-Bello ${ }^{5}$ \\ ${ }^{1}$ Center of Biophysics and Biochemistry, Venezuelan Institute for Scientific Research, IVIC, Caracas, \\ Venezuela \\ ${ }^{2}$ Department of Chemistry and Biochemistry, University of Colorado, UCB 215, Boulder, CO 80309, \\ USA \\ ${ }^{3}$ Amazonic Center for Research and Control of Tropical Diseases, CAICET, Puerto Ayuacucho, \\ Amazonas, Venezuela \\ ${ }^{4}$ Howard Hughes Medical Institute, 4000 Jones Bridge Road, Chevy Chase, MD 20815-6789, \\ USA \\ ${ }^{5}$ Department of Biology, University of Puerto Rico, Rio Piedras, San Juan, PR 00931, \\ Puerto Rico
}

\begin{abstract}
The oral microbiota plays an important role in buccal health and in diseases such as periodontitis and meningitis. The study of the human oral bacteria has so far focused on subjects from Western societies, while little is known about subjects from isolated communities. This work determined the composition of the oral mucosa microbiota from six Amazon Amerindians, and tested a sample preservation alternative to freezing. Paired oral swabs were taken from six adults of Guahibo ethnicity living in the community of Platanillal, Amazonas State, Venezuela. Replicate swabs were preserved in liquid nitrogen and in Aware Messenger fluid (Calypte). Buccal DNA was extracted, and the $\mathrm{V} 2$ region of the $16 \mathrm{~S}$ rRNA gene was amplified and pyrosequenced. A total of 17214 oral bacterial sequences were obtained from the six subjects; these were binned into 1034 OTUs from 10 phyla, 30 families and 51 genera. The oral mucosa was highly dominated by four phyla: Firmicutes (mostly the genera Streptococcus and Veillonella), Proteobacteria (mostly Neisseria), Bacterioidetes (Prevotella) and Actinobacteria (Micrococcineae). Although the microbiota were similar at the phylum level, the Amerindians shared only $62 \%$ of the families and $23 \%$ of the genera with non-Amerindians from previous studies, and had a lower richness of genera (51 vs 177 reported in non-Amerindians). The Amerindians carried unidentified members of the phyla Bacteroidetes, Firmicutes and Proteobacteria and their microbiota included soil bacteria Gp1 (Acidobacteriaceae) and Xylanibacter (Prevotellaceae), and the rare genus Phocoenobacter (Pasteurellaceae). Preserving buccal swabs in the Aware Messenger oral fluid collection device substantially altered the bacterial composition in comparison to freezing, and therefore this method cannot be used to preserve samples for the study of microbial communities.
\end{abstract}

\section{INTRODUCTION}

The oral cavity is a complex habitat for bacteria in healthy subjects, although bacteria also play a causative role in periodontal diseases (Kroes et al., 1999). More than 600 different bacterial species have been described from the human oral cavity (Keijser et al., 2008; Nasidze et al., 2009a), but these studies have been limited to Caucasians

Abbreviation: OTU, operational taxonomic unit.

Three supplementary tables are available with the online version of this paper. from modern societies in developed countries. Modern lifestyles involve the use of medicines (including antibiotics), and specific dietary and oral hygiene habits, gum chewing, amalgam fillings, smoking, and other factors that might affect the oral microbiota. The full diversity of the human microbiota can therefore be assessed only by studies in diverse human populations.

Recent improvements in DNA sequencing technology (e.g. barcoded pyrosequencing on the Genome Sequencer FLX/ 454 Life Sciences platform) have enabled a dramatic increase in throughput via parallel in-depth analysis of 
many samples simultaneously (Hamady et al., 2008; Meyer et al., 2008). These techniques have been successfully applied to determine bacterial diversity in different human body habitats, including the oral cavity, intestine, vagina and skin (Bik et al., 2006; Fierer et al., 2008; Keijser et al., 2008; Grice et al., 2009; Nasidze et al., 2009a; Zaura et al., 2009; Domínguez-Bello et al., 2010; Ravel et al., 2010). In this work we describe the composition of the bacterial communities of the oral mucosa in South American Amerindians living in a remote village, less exposed to selective pressures of modern life, and also tested a chemical sample preservation method that provides an alternative to freezing, which might facilitate human microbiome studies in remote geographical locations.

\section{METHODS}

Subjects and samples. Six apparently healthy Amerindian adults (18 to 45 years old, five 5 females and one male) of Guahibo ethnicity were sampled in the community of Platanillal, Amazonas, Venezuela, in June 2008 (Table 1). Volunteers signed an informed consent and the protocol was approved by the IVIC Bioethical Committee. Use of samples without identifiers was approved by the IRB at UPR. The village lacks electricity and running water, toilets are latrines and the people practise personal hygiene at the river or with buckets of water transported from the river or from one of the village wells. We did not study the oral health of the subjects, but most adults had evidence of dental cavities.

Two oral swab samples were taken from each individual, rubbing the cheek oral mucosa with a swab, five to seven times on each side. The swabs were cut and the tips were immediately preserved either in cryovials immersed in liquid nitrogen, or, in order to test the efficacy of a preserving solution, in an Aware Messenger oral fluid collection device (Calypte Biomedical Corporation) at room temperature. The Aware Messenger oral fluid collection device is marketed for the collection, stabilization and transport of oral fluid specimens. Aware has been tested to preserve IgG-rich oral fluid (http://www.calypte com/aware-messenger.asp), but has not been tested for preservation of nucleic acids. After 3 weeks, when samples arrived at the laboratory, all swabs were kept in an ultralow-temperature freezer at $-80{ }^{\circ} \mathrm{C}$ until DNA extraction and analysis. We decided to test the Aware in the samples we were collecting from Amazonian peoples rather than in laboratory studies that would have required extra sequencing.

Table 1. Description of the Amerindian subjects included in this work

All the subjects were of Guahibo ethnicity.

\begin{tabular}{|lclc|}
\hline Amerindian subject & Sex & Village & Age (years) \\
\hline PA08-71 & F & San Pablito & 19 \\
PA08-72 & M & Platanillal & 18 \\
PA08-75 & F & Platanillal & 28 \\
PA08-77 & F & Platanillal & 19 \\
PA08-83 & F & Platanillal & 35 \\
PA08-88* & F & Platanillal & 45 \\
\hline
\end{tabular}

${ }^{*}$ Taking amoxicillin at the time of sampling
DNA extraction. DNA was extracted from the oral swabs using the MO BIO Power Soil DNA Isolation kit after the swabs were put in bead tubes with $60 \mu \mathrm{l}$ Solution $\mathrm{C} 1$. The tubes were incubated at $65{ }^{\circ} \mathrm{C}$ for $10 \mathrm{~min}$ and vortexed for $2 \mathrm{~min}$, followed by the procedures indicated by the manufacturer. Extracted DNA was stored at $-20{ }^{\circ} \mathrm{C}$.

PCR amplification of the V2 region of bacterial 16S rRNA genes. The $16 \mathrm{~S}$ rRNA gene was amplified using a primer set as described by Fierer et al. (2008). The forward primer (5'-GCC TTG CCA GCC CGC TCA GTC AGA GTT TGA TCC TGG CTC AG-3') contained the 454 Life Sciences primer B sequence, the broadly conserved bacterial primer 27F, and a two-base linker sequence ('TC'). The reverse primer (5'-GCC TCC CTC GCG CCA TCA GNN NNN NNN NNN NCA TGC TGC CTC CCG TAG GAG T-3') contained the 454 Life Sciences primer A sequence, a unique $12 \mathrm{nt}$ error-correcting Golay barcode used to tag each PCR product (designated by NNNNNNNNNNNN), the broad-range bacterial primer 338R, and a ' $\mathrm{CA}$ ' linker sequence inserted between the barcode and the rRNA primer. PCRs were carried out in triplicate $25 \mu \mathrm{l}$ reactions with $0.6 \mu \mathrm{M}$ forward and reverse primers, $3 \mu \mathrm{l}$ template DNA, and $1 \times$ HotMasterMix (5 PRIME). All PCRs were prepared using certified DNA-free PCR water (MO BIO) in a PCR hood UV irradiated for at least $30 \mathrm{~min}$ before use. Thermal cycling consisted of initial denaturation at $94{ }^{\circ} \mathrm{C}$ for 3 min followed by 35 cycles of denaturation at $94{ }^{\circ} \mathrm{C}$ for $45 \mathrm{~s}$, annealing at $50{ }^{\circ} \mathrm{C}$ for $30 \mathrm{~s}$, and extension at $72{ }^{\circ} \mathrm{C}$ for $90 \mathrm{~s}$, with a final extension of $10 \mathrm{~min}$ at $72{ }^{\circ} \mathrm{C}$. Amplicons were cleaned using the UltraClean-htp 96-well PCR Clean-up kit (MO BIO) and DNA concentrations were measured using the Quant-iT PicoGreen dsDNA reagent and kit (Invitrogen). Following quantification, cleaned amplicons were combined in equimolar ratios into a single tube, and DNA was precipitated on ice for $45 \mathrm{~min}$ after the addition of $5 \mathrm{M} \mathrm{NaCl}(0.2 \mathrm{M}$ final concentration) and then 2 vols ice-cold $100 \%$ ethanol. The precipitated DNA was centrifuged at $7800 \mathrm{~g}$ for $40 \mathrm{~min}$ at $4{ }^{\circ} \mathrm{C}$, and the resulting pellet was washed with an equal volume of ice-cold $70 \%$ ethanol and centrifuged again at $7800 \mathrm{~g}$ for $20 \mathrm{~min}$ at $4{ }^{\circ} \mathrm{C}$. The supernatant was removed and the pellet was air-dried for $10 \mathrm{~min}$ at room temperature and resuspended in nuclease-free water (MO BIO).

Pyrosequencing and sequence analysis. Pyrosequencing was carried out on a 454 Life Sciences Genome Sequencer FLX instrument (Roche) by the Environmental Genomics Core Facility at the University of South Carolina (Columbia). Sequences (200-250 nt) were processed and analysed as described by Hamady et al. (2008), and assigned to operational taxonomic units (OTUs) using cd-hit ( $\mathrm{Li}$ \& Godzik, 2006) with an identity cut off of $97 \%$.

Representative sequences were aligned using NAST (DeSantis et al., 2006a) and the Greengenes database (DeSantis et al., 2006b) with a minimum alignment length of 150 and a minimum identity of $75 \%$. A phylogenetic tree was inferred using Clearcut (Sheneman et al., 2006) with Kimura's two-parameter model. Taxonomy was assigned using the RDP classifier with a minimum support threshold of $60 \%$ (Wang et al., 2007) and the RDP taxonomic nomenclature.

Community comparisons. To determine the amount of bacterial diversity shared between two communities (beta diversity) we employed the UniFrac metric (Lozupone \& Knight, 2005; Lozupone et al., 2007). UniFrac distances are based on the fraction of branch length shared between two communities within a phylogenetic tree constructed from the 16S rRNA gene sequences from all communities being compared. A relatively small UniFrac distance implies that two communities are compositionally similar, containing lineages sharing a common evolutionary history. In unweighted UniFrac, only the presence or absence of lineages is considered. In weighted UniFrac, branch lengths are weighted based on the relative abundances of lineages within communities. 


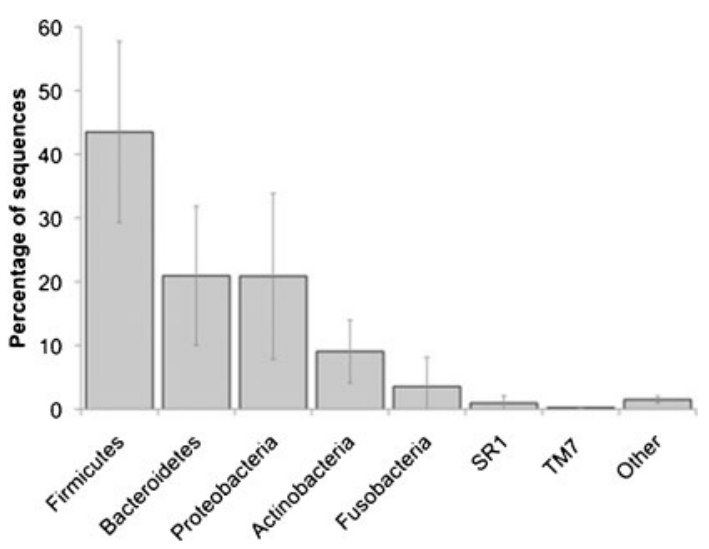

Fig. 1. Major bacterial phyla in the oral mucosa from six adult Guahibo Amerindians.

\section{RESULTS}

\section{Oral bacterial diversity}

A total of 17162 oral bacterial sequences were obtained, 8696 from the frozen swabs and 8466 from the Awarepreserved swabs. Unclassified sequences accounted for $0.3 \%$ of the total sequences. In total 1034 OTUs from 10 phyla were found in the oral mucosa community from samples preserved frozen or in Aware Messenger solution, although alpha diversity figures should be treated with caution as no denoising was performed on these samples. We found 693 OTUs from 10 phyla, 30 families and 51 genera, in the frozen oral mucosa swabs from the six Amerindians, $68.5 \%$ of which were unique (i.e. found in one person). Each Amerindian oral frozen sample had on average $220 \pm 39$ OTUs.
The bacterial sequences from the oral mucosa were highly dominated by four phyla (Fig. 1; see also Supplementary Table S1, available with the online version of this paper): Firmicutes (mostly the genera Streptococcus and Veillonella), Proteobacteria (mostly Neisseria), Bacterioidetes (Prevotella) and Actinobacteria (Micrococcineae). Other phyla included Fusobacteria, SR1, TM7 and other (Spirochaetes, Acidobacteria, Cyanobacteria, Tenericutes). The Amerindians carried unidentified members of the phyla Bacteroidetes, Firmicutes and Proteobacteria (Supplementary Table S1). No evidence of a substantial difference in the bacterial community between the subject taking amoxicillin (Table 1) and the other subjects was found.

\section{Effect of the alternative chemical sample preservation}

Preserving buccal swabs in the Aware Messenger oral fluid collection device altered the bacterial community composition relative to ultralow-temperature freezing (Table 2, Supplementary Table S2). UniFrac-based principal coordinates analysis indicated that sample storage in Aware Messenger solution shifted the bacterial community structure, with PC1 separating samples according to preservation method and explaining as much as $56.4 \%$ of the total sample variation (Fig. 2). Relative to frozen samples, Awarepreserved samples were enriched in Firmicutes (10 OTUs overrepresented), particularly in Streptococcus, while they had a reduced richness in 10 OTUs from the most dominant phyla, Bacterioidetes (Prevotella), Firmicutes (Veillonella) and Proteobacteria (Neisseria) (Table 2, Supplementary Table S2). Additionally, the Aware-preserved samples clustered together more tightly along PC2 (Fig. 2), suggesting lower variability, and that relative differences between individuals in this group are obscured by this preservation technique.

Table 2. Bacterial taxa that changed in Aware preservation compared to freezing

\begin{tabular}{|c|c|c|c|c|}
\hline $\begin{array}{l}\text { Direction of change } \\
\text { with Aware }\end{array}$ & Phylum & Order/family & Genus & $\begin{array}{c}\text { No. of OTUs } \\
\text { changed }\end{array}$ \\
\hline \multirow[t]{9}{*}{ Decrease } & Proteobacteria & Campylobacterales & & 1 \\
\hline & & Neisseriaceae & Neisseria & 2 \\
\hline & & Burkholderiales & & 1 \\
\hline & Firmicutes & Veillonellaceae & Veillonella & 3 \\
\hline & & Carnobacteriaceae & Granulicatella & 1 \\
\hline & Fusobacteria & Fusobacteriaceae & Fusobacterium & 1 \\
\hline & Bacterioidetes & Prevotellaceae & Prevotella & 2 \\
\hline & & Bacteroidales & & 2 \\
\hline & Actinobacteria & Actinomycetaceae & Actinomyces & 1 \\
\hline Increase & Firmicutes & Streptococcaceae & Streptococcus & 6 \\
\hline
\end{tabular}




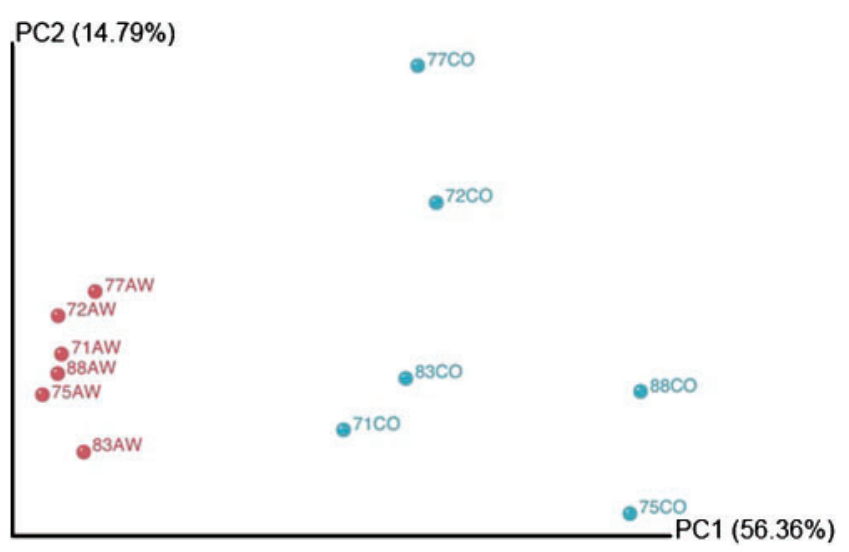

Fig. 2. Principal components analysis of bacterial communities from oral samples from six Guahibo Amerindians, preserved either in Aware Messenger solution (red) or in liquid nitrogen (blue). Beta diversity was analysed using the UniFrac metric, in which distances are based on the fraction of branch length shared between two communities within a phylogenetic tree constructed from the $16 \mathrm{~S}$ rRNA gene sequences from all communities being compared (Lozupone \& Knight, 2005; Lozupone et al., 2007).

\section{DISCUSSION}

This was a preliminary study conducted on only a few subjects, and at a sequencing depth sufficient to determine the general structure of the bacterial community at the supra-species level and inter-individual variability. The dominant phyla found in the oral mucosa of Amerindians were similar to those reported in buccal samples of nonAmerindians: Firmicutes, Proteobacteria, Actinobacteria (Huyghe et al., 2008; Lazarevic et al., 2009; Nasidze et al., 2009a, b; Zaura et al., 2009). With similar methodology to ours, in studies of non-Amerindians other authors found variable numbers of bacterial phyla in saliva (Table 3) Across-studies comparisons have to be made with caution, due to differences in sequencing efforts, methodologies and oral sites studied (Engelbrektson et al., 2010). When comparing bacteria from the oral mucosa in Amerindians with previous reports on non-Amerindians, we found similar numbers of families (30 in Amerindians and 33 in non-Amerindians), but with higher richness of genera in non-Amerindians (177) than in Amerindians (51) (Supplementary Table S3). Amerindians shared only $62 \%$ of the families and $23 \%$ of the genera with nonAmerindians (Fig. 3).

The Amerindians carried marker bacteria that were not reported in previous studies (Supplementary Table S3), such as soil bacteria Gp1 (Acidobacteriaceae) (Ausec et al., 2009; Castro et al., 2010), and the xylanolytic Xylanibacter (Prevotellaceae) (Ueki et al., 2006), as well as Phocoenobacter (Pasteurellaceae), first described in a porpoise (Foster et al., 2000), in addition to unidentified members of the phyla Bacteroidetes, Firmicutes and Proteobacteria.

Several methods, such as cooling, freezing, fixing, drying, freeze-drying and enzyme inhibitors, have been used to preserve samples (Brambilla et al., 1992). Freezing is the most widely used sample preservation method for molecular studies, but it can complicate the logistics of sampling in remote settings. Sample preservation devices have been developed to replace freezing, including FTA paper (Whatman), RNeasy Protect Saliva Mini kit (Qiagen) and Aware Messenger solution. None of these devices had to our knowledge been evaluated in terms of preservation of sample DNA, and this study demonstrates that at least the Aware Messenger is not a good method for preserving nucleic acids if the goal is to perform a community-level analysis with stored DNA. However, Aware Messenger apparently selected for some OTUs that were

Table 3. Recent studies of human oral bacteria using culture-independent methods

\begin{tabular}{|c|c|c|c|c|c|}
\hline Study & Subjects/lifestyle/sample & No. of subjects & OTU level & $\begin{array}{c}\text { No. of } \\
\text { bacterial } \\
\text { phyla }\end{array}$ & Method \\
\hline Paster et al. (2001) & Caucasian/urban/subgingival plaque & $\begin{array}{l}5 \text { healthy, } 26 \\
\text { diseased }\end{array}$ & 415 species & 9 & PCR/cloning \\
\hline Nasidze et al. (2009a) & Caucasian/urban/saliva & 120 healthy & 101 genera & 13 & \\
\hline Bik et al. (2010) & $\begin{array}{l}6 \text { Caucasian, } 1 \text { Afro-American, } 2 \text { Chinese, } \\
1 \text { Indian/urban/oral tissues and gingiva }\end{array}$ & 10 healthy & 247 species & 9 & \\
\hline Huyghe et al. (2008) & Caucasian/urban/gingival fluid & 2 healthy & 9 genera & 16 & Microarray \\
\hline Lazarevic et al. (2009) & Caucasian/urban/saliva and oropharyngeal & 3 healthy & 135 genera (V5) & 12 & Illumina \\
\hline Keijser et al. (2008) & Caucasian/urban/saliva & 71 healthy & $\begin{array}{l}3621 \text { species in } 31 \\
\text { genera }(\mathrm{V} 6)\end{array}$ & 22 & 454 pyrosequencing \\
\hline Nasidze et al. (2009b) & Caucasian/urban/saliva & 12 healthy & 79 genera $(\mathrm{V} 1, \mathrm{~V} 2)$ & 12 & \\
\hline Zaura et al. (2009) & $\begin{array}{l}\text { Caucasian/urban/dental surfaces, cheek, } \\
\text { hard palate, tongue and saliva }\end{array}$ & 3 healthy & 78 genera $(\mathrm{V} 5, \mathrm{~V} 6)$ & 8 & \\
\hline This study & Amerindian/rural/oral mucosa & 6 healthy & $\begin{array}{l}693 \text { species in } 51 \\
\text { genera (V2) }\end{array}$ & 10 & \\
\hline
\end{tabular}


(a)

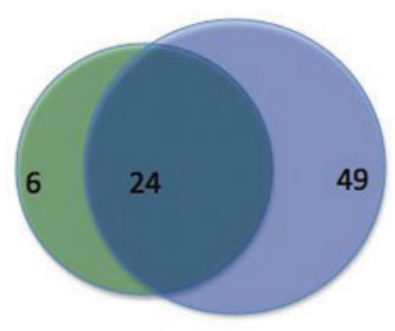

(b)

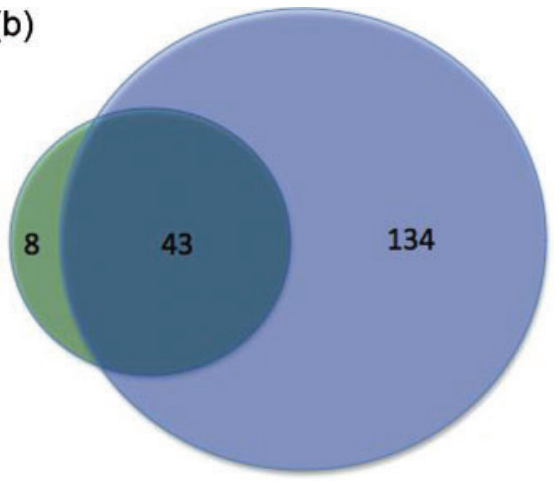

Fig. 3. Venn diagram of the numbers of shared and exclusive oral bacterial families (a) and genera (b) in Amerindians (green circles) and non-Amerindians (violet circles). undetected in the frozen swabs, such as Lactobacillales, Lactobacillus, Dialister and Ruminococcaceae (Firmicutes), Actinomycetales and Bifidobacteriaceae (Acidobacteria), although these may have been present in the Aware solution before adding the sample. However, if present in the Aware solution, we would expect the same species to have appeared in all samples preserved in Aware, which was not the case (Supplementary Table S2). In addition to DNA preservation, the Aware solution might have altered the growth capabilities of bacteria, enriching species that were able to survive or grow better. Aware Messenger solution is thus not a suitable alternative to freezing, since it affects the composition of the original sample relative to freezing.

\section{ACKNOWLEDGEMENTS}

We acknowledge Noah Fierer for assistance with the sequencing, and Calypte Corporation for the donation of the Aware Messenger solution. The project was partially financed by IVIC and SACAICET, Venezuela.

\section{REFERENCES}

Ausec, L., Kraighera, B. \& Mandic-Mulec, I. (2009). Differences in the activity and bacterial community structure of drained grassland and forest peat soils. Soil Biol Biochem 41, 1874-1881.

Bik, E. M., Eckburg, P. B., Gill, S. R., Nelson, K. E., Purdom, E. A., Francois, F., Perez-Perez, G., Blaser, M. J. \& Relman, D. A. (2006). Molecular analysis of the bacterial microbiota in the human stomach. Proc Natl Acad Sci U S A 103, 732-737.

Bik, E. M., Long, C. D., Armitage, G. C., Loomer, P., Emerson, J., Mongodin, E. F., Nelson, K. E., Gill, S. R., Fraser-Liggett, C. M. \& Relman, D. A. (2010). Bacterial diversity in the oral cavity of 10 healthy individuals. ISME J 4, 962-974.

Brambilla, E., Strohmenger, L. \& Vogel, G. (1992). The effect of storage in liquid nitrogen on the isolation of oral yeasts in human saliva. Arch Oral Biol 37, 237-239.

Castro, H. F., Classen, A. T., Austin, E. E., Norby, R. J. \& Schadt, C. W. (2010). Soil microbial community responses to multiple experimental climate change drivers. Appl Environ Microbiol 76, 999-1007.

DeSantis, T. Z., Jr, Hugenholtz, P., Keller, K., Brodie, E. L., Larsen, N., Piceno, Y. M., Phan, R. \& Andersen, G. L. (2006a). NAST: a multiple sequence alignment server for comparative analysis of 16S rRNA genes. Nucleic Acids Res 34, W394-W399.
DeSantis, T. Z., Hugenholtz, P., Larsen, N., Rojas, M., Brodie, E. L., Keller, K., Huber, T., Dalevi, D., Hu, P. \& Andersen, G. L. (2006b). Greengenes, a chimera-checked 16S rRNA gene database and workbench compatible with ARB. Appl Environ Microbiol 72, 5069-5072.

Domínguez-Bello, M. G., Costello, E. K., Contreras, M., Magris, M., Hidalgo, G., Fierer, N. \& Knight, R. (2010). Delivery mode shapes the acquisition and structure of the initial microbiota across multiple body habitats in newborns. Proc Natl Acad Sci U S A 107, 1197111975.

Engelbrektson, A., Kunin, V., Wrighton, K. C., Zvenigorodsky, N., Chen, F., Ochman, H. \& Hugenholtz, P. (2010). Experimental factors affecting PCR-based estimates of microbial species richness and evenness. ISME J 4, 642-647.

Fierer, N., Hamady, M., Lauber, C. L. \& Knight, R. (2008). The influence of sex, handedness, and washing on the diversity of hand surface bacteria. Proc Natl Acad Sci U S A 105, 17994-17999.

Foster, G., Ross, H. M., Malnick, H., Willems, A., Hutson, R. A., Reid, R. J. \& Collins, M. D. (2000). Phocoenobacter uteri gen. nov., sp. nov., a new member of the family Pasteurellaceae Pohl (1979) 1981 isolated from a harbour porpoise (Phocoena phocoena). Int J Syst Evol Microbiol 50, 135-139.

Grice, E. A., Kong, H. H., Conlan, S., Deming, C. B., Davis, J., Young, A.C, NISC Comparative Sequencing Program, Bouffard, G. G., Blakesley, R. W. \& other authors (2009). Topographical and temporal diversity of the human skin microbiome. Science 324, $1190-1192$.

Hamady, M., Walker, J. J., Harris, J. K., Gold, N. J. \& Knight, R. (2008). Error-correcting barcoded primers allow hundreds of samples to be pyrosequenced in multiplex. Nat Methods 5, 235-237.

Huyghe, A., Francois, P., Charbonnier, Y., Tangomo-Bento, M., Bonetti, E. J., Paster, B. J., Bolivar, I., Baratti-Mayer, D. \& Pittet, D. (2008). Novel microarray design strategy to study complex bacterial communities. Appl Environ Microbiol 74, 1876-1885.

Keijser, B. J., Zaura, E., Huse, S. M., van der Vossen, J. M., Schuren, F. H., Montijn, R. C., ten Cate, J. M. \& Crielaard, W. (2008). Pyrosequencing analysis of the oral microflora of healthy adults. J Dent Res 87, 1016-1020.

Kroes, I., Lepp, P. W. \& Relman, D. A. (1999). Bacterial diversity within the human subgingival crevice. Proc Natl Acad Sci U S A 96, 14547-14552.

Lazarevic, V., Whiteson, K., Huse, S., Hernandez, D., Farinelli, L., Osterås, M., Schrenzel, J. \& François, P. (2009). Metagenomic study of the oral microbiota by Illumina high-throughput sequencing. J Microbiol Methods 79, 266-271.

Li, W. Z. \& Godzik, A. (2006). Cd-hit: a fast program for clustering and comparing large sets of protein or nucleotide sequences. Bioinformatics 22, 1658-1659. 
Lozupone, C. \& Knight, R. (2005). UniFrac: a new phylogenetic method for comparing microbial communities. Appl Environ Microbiol 71, 8228-8235.

Lozupone, C. A., Hamady, M., Kelley, S. T. \& Knight, R. (2007). Quantitative and qualitative beta diversity measures lead to different insights into factors that structure microbial communities. Appl Environ Microbiol 73, 1576-1585.

Meyer, M., Stenzel, U. \& Hofreiter, M. (2008). Parallel tagged sequencing on the 454 platform. Nat Protoc 3, 267-278.

Nasidze, I., Li, J., Quinque, D., Tang, K. \& Stoneking, M. (2009a). Global diversity in the human salivary microbiome. Genome Res 19, 636-643.

Nasidze, I., Quinque, D., Li, J., Li, M., Tang, K. \& Stoneking, M. (2009b). Comparative analysis of human saliva microbiome diversity by barcoded pyrosequencing and cloning approaches. Anal Biochem 391, 64-68.

Paster, B. J., Boches, S. K., Galvin, J. L., Ericson, R. E., Lau, C. N., Levanos, V. A., Sahasrabudhe, A. \& Dewhirst, F. E. (2001). Bacterial diversity in human subgingival plaque. J Bacteriol 183, 3770-3783.
Ravel, J., Gajer, P., Abdo, Z., Schneider, G. M., Koenig, S. S., McCulle, S. L., Karlebach, S., Gorle, R., Russell, J. \& other authors (2010). Microbes and Health Sackler Colloquium: vaginal microbiome of reproductive-age women. Proc Natl Acad Sci U S A Aug 19 [Epub ahead of print].

Sheneman, L., Evans, J. \& Foster, J. A. (2006). Clearcut: a fast implementation of relaxed neighbor joining. Bioinformatics 22, 28232824.

Ueki, A., Akasaka, H., Suzuki, D., Hattori, S. \& Ueki, K. (2006). Xylanibacter oryzae gen. nov., sp. nov., a novel strictly anaerobic, Gramnegative, xylanolytic bacterium isolated from rice-plant residue in flooded rice-field soil in Japan. Int J Syst Evol Microbiol 56, 2215-2221.

Wang, Q., Garrity, G. M., Tiedje, J. M. \& Cole, J. R. (2007). Naive Bayesian classifier for rapid assignment of rRNA sequences into the new bacterial taxonomy. Appl Environ Microbiol 73, 5261-5267.

Zaura, E., Keijser, B. J., Huse, S. M. \& Crielaard, W. (2009). Defining the healthy "core microbiome" of oral microbial communities. BMC Microbiol 9, 259.

Edited by: P. W. O'Toole 